\title{
Undamped Nonlinear Consensus Using Integral Lyapunov Functions
}

\author{
Martin Andreasson* ${ }^{* \dagger}$, Dimos V. Dimarogonas* and Karl H. Johansson* \\ ${ }^{*}$ ACCESS Linnaeus Center, KTH Royal Institute of Technology, Stockholm, Sweden.
}

\begin{abstract}
This paper analyzes a class of nonlinear consensus algorithms where the input of an agent can be decoupled into a product of a gain function of the agents own state, and a sum of interaction functions of the relative states of its neighbors. We prove the stability of the protocol for both single and double integrator dynamics using novel Lyapunov functions, and provide explicit formulas for the consensus points. The results are demonstrated through simulations of a realistic example within the framework of our proposed consensus algorithm.
\end{abstract}

\section{INTRODUCTION}

The consensus problem has received a tremendous research interest over the past years. The coordination of autonomous agents based solely on local interactions and decentralized control algorithms [20], has applications in formation-control [9], flocking [14], [24] and rendezvous [4] amongst others.

While most research effort has been on analyzing linear consensus, many applications of consensus protocols are inherently nonlinear. This paper contributes to a deeper insight in nonlinear consensus by considering a class of nonlinear consensus protocols where the input of an agent can be decoupled into a product of a positive gain function of the agents own state, and a sum of interaction functions of its neighbors relative states. Nonlinear interacton functions are a well-studied problem [17], [5], [10], with applications in consensus while preserving connectedness [11], [8] and collision avoidance [18], [8], [23]. Sufficient conditions for the convergence of nonlinear protocols for first-order integrator dynamics are given in [1] and in [13] for a multidimensional state-space.

Consensus on a general function value through nonlinear gain functions was first introduced in [20] as $\chi$-consensus, and a solution to the $\chi$-consensus problem was presented in [6]. $\chi$-consensus has applications for instance in weighted power mean consensus [6], [7], [3]. The literature on $\chi$ consensus has been focused on agents with single integrator dynamics. However, as we show later, the results can be generalized to also hold for second-order integrator dynamics.

Consensus protocols where the input of an agent can be separated into a product of a positive function of the agents

This work was supported in part by the European Commission, the Swedish Research Council and the Knut and Alice Wallenberg Foundation. The $2^{\text {nd }}$ author is also affiliated with the Centre for Autonomous Systems at KTH and is supported by the VR 2009-3948 grant. $\dagger$ Corresponding author. E-mail: mandreas@kth.se own state were studied in [3] for single integrator dynamics. [2] extended the results to switching communication topologies, where the communication graph always remains connected. Linear consensus for double integrator dynamics were studied in detail in [22] for undirected as well as for directed communication. Necessary and sufficient conditions for consensus with double integrator dynamics and directed communication topology were given in [25]. Extensions to a certain type of nonlinear control law were made in [21]. The more general model where both the gains and the interaction functions are nonlinear is however not mentioned, and to our best knowledge this is the first work addressing the more general model. [16] studies position consensus for agents with double integrator dynamics under a class of nonlinear interaction functions and nonlinear velocity-damping. In contrast to this, we consider agents with single integrator dynamics and undamped double integrator dynamics.

There main contributions of this paper are twofold. First, we derive explicit bounds on the convergence rate for a class of nonlinear consensus protocols for first-order dynamics, using a novel Lyapunov function which penalizes the sum of weighted integrals of the deviations from the equilibrium states of the agents. A second contribution of this paper is the generalization of previous results on linear consensus algorithms for double integrator dynamics to nonlinear algorithms. Here we borrow the concept of the novel Lyapunov function, penalizing the sum of integrals of the deviations from the equilibrium velocities of the agents, but add a penalty also on an integral of the disagreement over the communication links.

The rest of this paper is organized as follows. In section II we investigate nonlinear consensus for single integrator dynamics, where we bound the convergence rate from below using a novel Lyapunov function. We also derive necessary and sufficient conditions for consensus under switching topologies. In section III we derive necessary and sufficient conditions for consensus under double integrator dynamics. In section IV we demonstrate our results by a comprehensive example, followed by some concluding remarks in section $\mathrm{V}$.

\section{CONSENSUS FOR SINGLE INTEGRATOR DYNAMICS}

In this section we review the most important results derived in [3]. We first make some definitions used later on in this paper. Let $\mathcal{G}$ be a graph. Denote by $\mathcal{V}=\{1, \ldots, n\}$ the vertex set of $\mathcal{G}$, and by $\mathcal{E}=\{1, \ldots, m\}$ the edge set of $\mathcal{G}$. 
Let $\mathcal{N}_{i}$ be the set of neighboring nodes to $i$. Denote by $\mathcal{B}=$ $\mathcal{B}(\mathcal{G})$ the adjacency matrix of $\mathcal{G}$, and let $\mathcal{L}$ be the Laplacian matrix of $\mathcal{G}$. We will denote the position of agent $i$ as $x_{i}$, and its velocity as $v_{i}$, and collect them into column vectors $x=\left(x_{1}, \ldots, x_{n}\right)^{T}, v=\left(v_{1}, \ldots, v_{n}\right)^{T}$. A function $f(\cdot)$ with domain $\mathcal{X}$ is said to be Lipschitz (continuous) if there exists $K \in \mathbb{R}^{+}: \forall x, y \in \mathcal{X}:\|f(x)-f(y)\| \leq K \cdot\|x-y\|$.

\section{A. Directed graphs}

We consider the following nonlinear first-order consensus protocol where each node $i$ applies the control signal:

$$
\dot{x}_{i}=u_{i}=-\gamma_{i}\left(x_{i}\right) \sum_{j \in \mathcal{N}_{i}} \alpha_{i j}\left(x_{i}-x_{j}\right) .
$$

We make the following assumptions on the gain and interaction functions.

Assumption 1. $\gamma_{i}$ is continuous and $\gamma_{i}(x)>0 \quad \forall i \in \mathcal{V}$

Assumption 2. $\alpha_{i j}(\cdot)$ is Lipschitz continuous $\forall i \in \mathcal{V}, \forall(i, j) \in \mathcal{E}$, and furthermore: $x \cdot \alpha_{i j}(x)>0 \quad \forall x \neq 0, \alpha_{i j}(0)=0 \forall(i, j) \in \mathcal{E}$.

Assumption 2 ensures that $\alpha_{i j}(\cdot)$ is an odd function. We are now ready to state the following result.

Theorem 1. Given $n$ agents obeying the agreement protocol (1) with $\alpha_{i j}$ and $\gamma_{i}$ satisfying assumptions 2 and 1 respectively, then for any initial condition $x(0)$, the agents converge to an agreement point $x^{*}$, satisfying $\min _{i} x_{i}(0) \leq$ $x^{*} \leq \max _{i} x_{i}(0)$ if and only if the underlying communication graph $\mathcal{G}$ contains a directed spanning tree. Furthermore if all directed spanning trees contained in $\mathcal{G}$ have the same root node, then $x^{*}=x_{r}(0)$.

Before giving the proof, we need the following lemma.

Lemma 2. Let $\mathcal{G}$ be a directed graph not containing a directed spanning tree. Then there exist strongly connected components $\mathcal{G}_{1}, \mathcal{G}_{2} \subset \mathcal{G}$ with $\mathcal{G}_{1} \cap \mathcal{G}_{2}=\emptyset$ such that there exist no incoming edges to $\mathcal{G}_{1}$ from $\mathcal{G} \backslash \mathcal{G}_{1}$ and no incoming edges to $\mathcal{G}_{2}$ from $\mathcal{G} \backslash \mathcal{G}_{2}$.

Proof: Let $\mathcal{G}$ be a directed graph not containing a directed spanning tree. Assume that there do not exist strongly connected components $\mathcal{G}_{1}, \mathcal{G}_{2} \subset \mathcal{G}$ with $\mathcal{G}_{1} \cap \mathcal{G}_{2}=\emptyset$ such that there exists no incoming edges to $\mathcal{G}_{1}$ from $\mathcal{G} \backslash \mathcal{G}_{1}$ and no incoming edges to $\mathcal{G}_{2}$ from $\mathcal{G} \backslash \mathcal{G}_{2}$. There exist two possibilities. In case 1 there exists only one strongly connected component $\mathcal{G}_{1}$ with no incoming edges, and in case 2 there does not exist any strongly connected component with no incoming edges. We now show only case 1 is possible, implying that $\mathcal{G}$ must contain a directed spanning tree.

Case 1: Denote by $\mathcal{G}_{1}, \ldots, \mathcal{G}_{k}$ the disjoint strongly connected components of $\mathcal{G}$, and assume without loss of generality that $\mathcal{G}_{1}, \ldots, \mathcal{G}_{k}$ are maximal. Let $\mathcal{G}_{1}$ be the only component with no incoming edges. Consider any component $\mathcal{G}_{i_{1}}, i_{1} \neq$ 1. Since $\mathcal{G}_{i_{1}}$ by assumption has an incoming edge from say component $\mathcal{G}_{i_{2}}$, there exists a directed path from any node in $\mathcal{G}_{i_{2}}$ to any node in $\mathcal{G}_{i_{1}}$. By applying the same argument recursively, since $\mathcal{G}$ is finite, there is either a directed path from $\mathcal{G}_{1}$ to $\mathcal{G}_{i_{1}}$, or a loop containing $\mathcal{G}_{i_{1}}$. But the existence of a loop would imply that any node on the loop is reachable from any other node on the loop, since the components are assumed to be strongly connected. But this would contradict the assumption of $\mathcal{G}_{1}, \ldots, \mathcal{G}_{k}$ being maximal. Thus there exists a path from any node in $\mathcal{G}_{1}$ to any node in $\mathcal{G}_{i_{1}}$. Since $\mathcal{G}_{i_{1}}$ was arbitrary there exists a path from any node in $\mathcal{G}_{1}$ to any other node in $\mathcal{G}$, implying that $\mathcal{G}$ contains a directed spanning tree, with root node in $\mathcal{G}_{1}$.

Case 2: We show that $\mathcal{G}$ must contain at least one strongly connected component, and that this case may be excluded. Denote by $\mathcal{G}_{1}, \ldots, \mathcal{G}_{k}$ the disjoint strongly connected components of $\mathcal{G}$, and assume without loss of generality that $\mathcal{G}_{1}, \ldots, \mathcal{G}_{k}$ are maximal. Consider any component, say $\mathcal{G}_{i_{1}}$. Like in the previous case there is a directed path from any node in $\mathcal{G}_{i_{2}}$ to any node in say $\mathcal{G}_{i_{1}}$. We can apply the same argument recursively, and since $\mathcal{G}$ is finite and by assumption there exists no component with no incoming edge, the path must eventually form a cycle containing at least two components. But this would imply that the components in the cycle together form a strongly connected component, violating our assumption that $\mathcal{G}_{1}, \ldots, \mathcal{G}_{k}$ are maximal.

Proof: (of theorem 1):

(Sufficiency:) The proof idea is similar to the one presented in [15], by using a Lyapunov function based on the convex hull of the agents' states. Consider the candidate Lyapunov function $V(x)=x_{i_{1}}-x_{i_{2}} \geq 0, x_{i_{1}} \in \mathcal{S}_{1}, x_{i_{2}} \in \mathcal{S}_{2}$, where:

$$
\begin{aligned}
& \mathcal{S}_{1}=\left\{i_{1}: x_{i_{1}}=\max _{i} x_{i}, \min _{k \in \mathcal{N}_{i_{1}}} x_{k}<x_{i_{1}}\right\} \\
& \mathcal{S}_{2}=\left\{i_{2}: x_{i_{2}}=\min _{i} x_{i}, \max _{k \in \mathcal{N}_{i_{2}}} x_{k}>x_{i_{2}}\right\}
\end{aligned}
$$

Assume that consensus is not reached, i.e. $V(x)>0$. It is now shown that $\mathcal{S}_{1} \cup \mathcal{S}_{2} \neq \emptyset$. Assume for the sake of contradiction that $\mathcal{S}_{1} \cup \mathcal{S}_{2}=\emptyset$. Let $\mathcal{M}_{1}=\left\{k: x_{k}=\max _{i} x_{i}\right\}$ and $\mathcal{M}_{2}=\left\{k: x_{k}=\min _{i} x_{i}\right\}$. If $k_{1} \in \mathcal{M}_{1}$ we must have $\mathcal{N}_{k_{1}} \in \mathcal{M}_{1}$ and if $k_{2} \in \mathcal{M}_{2}$ we must have $\mathcal{N}_{k_{2}} \in \mathcal{M}_{2}$, since otherwise either $S_{1}$ or $S_{2}$ would be non-empty. Since $\mathcal{G}$ by assumption contains a directed spanning tree, there exists a root node $k^{*}$ such that there exists a path from $k^{*}$ to any node $i \in \mathcal{V}$. Three distinct cases exist, which we show all contradict our assumption that $\mathcal{S}_{1} \cup \mathcal{S}_{2}=\emptyset$ :

Case 1: $k^{*} \in \mathcal{M}_{1}$. There exists a path from $k^{*}$ to $\mathcal{M}_{2}$. Thus for at least one node $i \in \mathcal{M}_{2} \exists j \in \mathcal{N}_{i}: j \notin \mathcal{M}_{2}$, which contradicts $\mathcal{S}_{1} \cup \mathcal{S}_{2}=\emptyset$.

Case 2: $k^{*} \in \mathcal{M}_{2}$. There exists a path from $k^{*}$ to $\mathcal{M}_{1}$. Thus for at least one node $i \in \mathcal{M}_{1} \exists j \in \mathcal{N}_{i}: j \notin \mathcal{M}_{1}$, which again contradicts $\mathcal{S}_{1} \cup \mathcal{S}_{2}=\emptyset$.

Case 3: $k^{*} \in \mathcal{V} \backslash\left(\mathcal{M}_{1} \cup \mathcal{M}_{2}\right)$. There exists a path from $k^{*}$ to $\mathcal{M}_{1}$, and a path from $k^{*}$ to $\mathcal{M}_{2}$. This implies there exist at least two nodes, $i_{1} \in \mathcal{M}_{1}, i_{2} \in \mathcal{M}_{2}$ such that $\exists j \in \mathcal{N}_{i_{1}}: j \notin \mathcal{M}_{1}$ and $\exists j \in \mathcal{N}_{i_{2}}: j \notin \mathcal{M}_{2}$, which again 
contradicts $\mathcal{S}_{1} \cup \mathcal{S}_{2}=\emptyset$. Thus

$$
\begin{array}{r}
\frac{\mathrm{d} V(x(t))}{\mathrm{d} t}=\dot{x}_{i_{1}}-\dot{x}_{i_{2}}=\gamma_{i}\left(x_{i_{1}}\right) \sum_{j \in \mathcal{N}_{i_{1}}} \alpha_{i_{1} j}\left(x_{j}-x_{i_{1}}\right) \\
-\gamma_{i}\left(x_{i_{2}}\right) \sum_{j \in \mathcal{N}_{i_{2}}} \alpha_{i_{2} j}\left(x_{j}-x_{i_{2}}\right) \\
\leq \gamma_{i}\left(x_{i_{1}}\right) \alpha_{i_{1} i_{1}^{*}}\left(x_{i_{1}^{*}}-x_{i_{1}}\right)-\gamma_{i}\left(x_{i_{2}}\right) \alpha_{i_{2} i_{2}^{*}}\left(x_{i_{2}^{*}}-x_{i_{2}}\right)<0
\end{array}
$$

where $x_{i_{1}^{*}}=\operatorname{argmin}_{k \in \mathcal{N}_{i_{1}}} x_{k}$ and $x_{i_{2}^{*}}=\operatorname{argmax}_{k \in \mathcal{N}_{i_{2}}} x_{k}$. Furthermore $\dot{V}(x)=0 \Leftrightarrow x=x^{*} 1$. Thus the agents converge to a common value $x^{*}$. It is also clear that $x^{\min } \leq x^{*} \leq x^{\max }$.

(Necessity:) Assume that $\mathcal{G}$ contains no directed spanning tree. By lemma $2 \exists \mathcal{G}_{1}, \mathcal{G}_{2} \subset \mathcal{G}$ such that $\mathcal{G}_{1}, \mathcal{G}_{2}$ are disjoint and there exists no incoming edges to $\mathcal{G}_{1}$ from $\mathcal{G} \backslash \mathcal{G}_{1}$ and no incoming edges to $\mathcal{G}_{2}$ from $\mathcal{G} \backslash \mathcal{G}_{2}$. Let

$$
x_{i}(0)= \begin{cases}x_{0}^{1} & \forall i \in \mathcal{G}_{1} \\ x_{0}^{2} & \forall i \in \mathcal{G}_{2}\end{cases}
$$

where $x_{0}^{1} \neq x_{0}^{2}$. By (1) we have that $\dot{x}_{i}=0 \quad \forall i \in \mathcal{G}_{1} \cup \mathcal{G}_{2}$. Thus consensus cannot be reached.

\section{B. Linear interaction functions}

If the consensus protocol is modified in such a way that we remove the nonlinearity of $\alpha_{i j}(\cdot)$, we can make a stronger statement on the final consensus value based on the theory from linear consensus on directed communication graphs. Let the consensus protocol be given by

$$
\dot{x}=\gamma_{i}\left(x_{i}\right) \sum_{j \in N_{i}}\left(x_{j}-x_{i}\right)
$$

with $\gamma_{i}(\cdot)$ satisfying assumption 1 .

Theorem 3. Let $\mathcal{G}$ be connected. By [19] the Laplacian, $\mathcal{L}$, has a left eigenvector $e$, with $e_{i}>0$ such that $e^{T} \mathcal{L}=0$. Then the agents converge to a common point $x^{*}$, satisfying $\sum_{i \in \mathcal{V}} e_{i} \int_{0}^{x_{i}^{0}} \frac{1}{\gamma_{i}(y)} \mathrm{d} y=\int_{0}^{x^{*}} \sum_{i \in \mathcal{V}} e_{i} \frac{1}{\gamma_{i}(y)} \mathrm{d} y$

Proof: Convergence follows by Theorem 1. Consider the quantity $E(x)=\sum_{i \in \mathcal{V}} e_{i} \int_{0}^{x_{i}} \frac{1}{\gamma_{i}(y)} \mathrm{d} y$. Differentiating with respect to time yields

$$
\begin{aligned}
\frac{\mathrm{d} E(x(t))}{\mathrm{d} t} & =\sum_{i \in \mathcal{V}} \frac{\partial E(x)}{\partial x_{i}} \frac{\partial x_{i}}{\partial t} \\
& =\sum_{i \in \mathcal{V}} e_{i} \frac{1}{\gamma_{i}\left(x_{i}\right)} \gamma_{i}\left(x_{i}\right) \sum_{j \in N_{i}}\left(x_{j}-x_{i}\right) \\
& =\sum_{i \in \mathcal{V}} e_{i} \sum_{j \in N_{i}}\left(x_{j}-x_{i}\right)=e^{T} \mathcal{L} x=0
\end{aligned}
$$

Hence $E\left(x^{0}\right)=E\left(x^{*}\right)$, which concludes the proof.

\section{Undirected graphs}

Assume now that the communication topology is undirected, which is formalized in the following assumption.

Assumption 3. $\alpha_{i j}(\cdot)$ is Lipschitz continuous $\forall i \in$ $\mathcal{V}, \forall \quad(i, j) \in \mathcal{E}$, and furthermore: $\alpha_{i j}(-y)=$ $-\alpha_{j i}(y) \quad \forall(i, j) \in \mathcal{E}, \forall y \in \mathbb{R}$ and $y \cdot \alpha_{i j}(y)>0 \quad \forall(i, j) \in$ $\mathcal{E}, \forall y \in \mathbb{R} \backslash\{0\}, \alpha_{i j}(0)=0$

We are now ready to state the main result of this section.

Theorem 4. Given $n$ agents obeying the agreement protocol (1) with $\alpha_{i j}$ and $\gamma_{i}$ satisfying assumptions 3 and 1 respectively, then the agents asymptotically converge to an agreement point $x^{*}$, uniquely determined by

$$
\sum_{i \in \mathcal{V}} \int_{0}^{x_{i}^{0}} \frac{1}{\gamma_{i}(y)} \mathrm{d} y=\int_{0}^{x^{*}} \sum_{i \in \mathcal{V}} \frac{1}{\gamma_{i}(y)} \mathrm{d} y
$$

for any set of initial conditions $x_{i}(0)=x_{i}^{0}$, if and only if the underlying communication graph $\mathcal{G}$ is connected.

Proof: (Sufficiency:) Consider the candidate Lyapunov function $V(x(t))=\sum_{i \in \mathcal{V}} \sum_{j \in \mathcal{N}_{i}} \int_{0}^{x_{i}-x_{j}} \alpha_{i j}(y) \mathrm{d} y>$ 0 iff $\bar{x} \neq 0$ where $\bar{x}=B^{T} x$. Differentiating with respect to time yields

$$
\dot{V}=\frac{\mathrm{d} V}{\mathrm{~d} t}=\frac{\partial V}{\partial \bar{x}} \frac{\partial \bar{x}}{\partial t}=\frac{\partial V}{\partial \bar{x}} \mathcal{B}^{T} \frac{\partial x}{\partial t} .
$$

Defining $\alpha(\bar{x})=\left[\alpha_{1}\left(\bar{x}_{1}\right), \ldots, \alpha_{m}\left(\bar{x}_{m}\right)\right]^{T}$, it is easily shown that: $\frac{\partial V(x(t))}{\partial \bar{x}}=\alpha(\bar{x})^{T}$. We write (1) in vector form as $\dot{x}=-\Gamma(x) B \alpha(\bar{x})$, where $x=$ $\left[x_{1}, \ldots, x_{n}\right]^{T}, \Gamma(x)=\operatorname{diag}\left(\left[\gamma_{1}\left(x_{1}\right), \ldots, \gamma_{n}\left(x_{n}\right)\right]\right)$. Hence $\dot{V}=-\alpha(\bar{x}) \mathcal{B}^{T} \Gamma(x) \mathcal{B} \alpha(\bar{x})=-\left\|\Gamma(x)^{\frac{1}{2}} \mathcal{B} \alpha(\bar{x})\right\|_{2}^{2} \leq 0$, and $\Gamma(x)^{\frac{1}{2}} \mathcal{B} \alpha(\bar{x})=0 \Leftrightarrow \mathcal{B} \alpha\left(\mathcal{B}^{T} x\right)=0 \Leftrightarrow \mathcal{B B}^{T} x=0$ by 3 . But this implies $x=x^{*} 1$ since $\mathcal{B B}^{T}=\mathcal{L}$, which for connected graphs $\mathcal{G}$ has a single zero eigenvalue, with 1 as corresponding eigenvector. Hence the agents converge to an agreement point $x_{i}=x^{*} \forall i$. The necessity part of the proof is trivial and omitted. Now consider the quantity $E(x)=\sum_{i \in \mathcal{V}} \int_{0}^{x_{i}} \frac{1}{\gamma_{i}(y)} \mathrm{d} y$. Differentiating with respect to time yields

$$
\begin{aligned}
& \frac{\mathrm{d} E(x(t))}{\mathrm{d} t}=\frac{\partial E(x(t))}{\partial x} \frac{\partial x}{\partial t} \\
= & -\left[\frac{1}{\gamma_{1}\left(x_{1}\right)}, \ldots, \frac{1}{\gamma_{n}\left(x_{n}\right)}\right] \Gamma(x) B \alpha(\bar{x})=-\mathbf{1}^{T} B \alpha(\bar{x})=0
\end{aligned}
$$

Hence $E(x)$ is invariant and the agreement point $x^{*}$ is given by (3). By assumption $1, E\left(x^{*}\right)$ is strictly increasing, and hence (3) admits a unique solution.

Note 1. The agreement protocol (1) has an intuitive physical interpretation. If we interpret $x_{i}$ as the temperature of the nodes, $\frac{1}{\gamma_{i}(\cdot)}$ can be seen as the temperature-dependent heat capacity of the nodes. Analogously, $\alpha_{i j}(\cdot)$ may be seen as the thermal conductivity of the links, being dependent on the heat flow in the link. The invariant quantity $E(x)$ is the total energy of the system, and the Lyapunov function $V(x)$ is the sum of the potential energies in the links.

\section{Bounded interaction functions}

We now make the following additional assumptions on the gain functions and the interaction functions:

Assumption 4. The interaction functions $\alpha_{i j}(\cdot)$ satisfy: $\underline{\alpha}_{i j} \cdot$ $=y \leq \alpha_{i j}(y) \quad \forall(i, j) \in \mathcal{E}, \forall y \in \mathbb{R}$, where $\underline{\alpha}_{i j} ; \in \mathbf{R}^{+}$. 
Assumption 4 imposes a uniform linear lower bound on the interaction functions. This is desirable in most applications, since having arbitrary small interaction functions would cause slow convergence. The following lemma shows that also $\gamma_{i}(\cdot)$ is uniformly bounded.

Lemma 5. $\gamma_{i}(x(t))$ is bounded by: $\underline{\gamma} \leq \gamma_{i}(x(t)) \leq \bar{\gamma} \quad \forall i \in$ $\mathcal{E}, \forall t \in \mathbb{R}^{+}$for some $\gamma, \bar{\gamma} ; \in \mathbf{R}^{+}$, depending only on the initial condition $x(0)$.

Proof: By the proof of theorem 1, $\max _{i} x_{i}$ is non-increasing while $\min _{i} x_{i}$ is non-decreasing. Hence $\min _{i} x_{i}(0) \leq x_{i}(t) \leq \max _{i} x_{i}(0) \forall i \in \mathcal{V}, \forall t \in \mathbb{R}^{+}$. Since a continuous function on a compact set attains its minimum and maximum, there exist $\underline{\gamma}$ and $\bar{\gamma}$ such that $\underline{\gamma} \leq \gamma_{i}(x(t)) \leq \bar{\gamma} \quad \forall i \in \mathcal{E}, \forall t \in \mathbb{R}^{+}$.

We now prove that the rate of convergence is bounded by an exponential function. Let: $\tilde{x}=\left[x_{i}-x^{*}, \ldots, x_{n}-x^{*}\right]^{T}$

Theorem 6. Given $n$ agents with initial condition $\tilde{x}(0)$ obeying (1) and satisfying assumption 4, the disagreement vector $\tilde{x}(t)$ satisfies $\|\tilde{x}(t)\|_{2} \leq \sqrt{\frac{\bar{\gamma}}{\underline{\gamma}}}\|\tilde{x}(0)\|_{2} e^{-\lambda_{2}\left(\mathcal{L}_{\underline{\alpha}}\right) \underline{\gamma} t}$, where $\lambda_{2}\left(\mathcal{L}_{\underline{\alpha}}\right)$ denotes the second smallest eigenvalue of $\mathcal{L}_{\underline{\alpha}}$.

Remark 1. Theorem 6 extends the results in [3]. Under certain conditions on $\alpha_{i j}(\cdot)$ and $\gamma_{i}(\cdot)$, the agents converge to an agreement point at least at exponential rate. The bound on the convergence speed relies heavily on a novel Lyapunov function, which penalizes a weighted integral of the deviation from the equilibrium state for each agent.

Proof: Consider the following candidate Lyapunov function:

$$
V(x(t))=\sum_{i \in \mathcal{V}} \int_{x^{*}}^{x_{i}} \frac{y-x^{*}}{\gamma_{i}(y)} \mathrm{d} y .
$$

It is easily verified that $V(x) \geq 0$ and $V(x)=0 \Leftrightarrow x=0$. Now consider the time derivative of $V(x)$ along trajectories of the closed loop system:

$$
\begin{aligned}
& \frac{\mathrm{d} V(x(t))}{\mathrm{d} t}=\sum_{i \in V} \frac{\partial V(x(t))}{\partial x_{i}} \frac{\partial x_{i}}{\partial t} \\
& =-\sum_{i \in \mathcal{V}} \frac{x_{i}-x^{*}}{\gamma_{i}\left(x_{i}\right)} \cdot \gamma_{i}\left(x_{i}\right) \sum_{j \in \mathcal{N}_{i}} \alpha_{i j}\left(x_{i}-x_{j}\right) \\
& =-\sum_{i \in \mathcal{V}} x_{i} \sum_{j \in \mathcal{N}_{i}} \alpha_{i j}\left(x_{i}-x_{j}\right)+\sum_{i \in \mathcal{V}} x^{*} \sum_{j \in \mathcal{N}_{i}} \alpha_{i j}\left(x_{i}-x_{j}\right) \\
& =-\frac{1}{2} \sum_{i \in \mathcal{V}} \sum_{j \in \mathcal{N}_{i}}\left(x_{i}-x_{j}\right) \alpha_{i j}\left(x_{i}-x_{j}\right) \\
& \leq-\frac{1}{2} \sum_{i \in \mathcal{V}} \sum_{j \in \mathcal{N}_{i}} \underline{\alpha}_{i j}\left(x_{i}-x_{j}\right)^{2} \\
& =-\frac{1}{2} \sum_{i \in \mathcal{V}} \sum_{j \in \mathcal{N}_{i}} \underline{\alpha}_{i j}\left[\left(x_{i}-x^{*}\right)-\left(x_{j}-x^{*}\right)\right]^{2} \\
& =-\sum_{i \in \mathcal{V}}\left(x_{i}-x^{*}\right)^{2} \sum_{j \in \mathcal{N}_{i}} \underline{\alpha}_{i j}+\sum_{i \in \mathcal{V}} \sum_{j \in \mathcal{N}_{i}} \underline{\alpha}_{i j}\left(x_{i}-x^{*}\right)\left(x_{j}-x^{*}\right) \\
& =-\tilde{x}^{T} \mathcal{L}_{\underline{\alpha}} \tilde{x}
\end{aligned}
$$

where $\mathcal{L}_{\underline{\alpha}}$ is the weighted Laplacian given by

$$
\mathcal{L}_{\underline{\alpha}}=\left[\begin{array}{ccc}
\sum_{j \in \mathcal{V}} k_{1 j} \underline{\alpha}_{1 j} & \ldots & -k_{1 n} \underline{\alpha}_{1 n} \\
-k_{21} \underline{\alpha}_{21} & \ldots & -k_{2 n} \underline{\alpha}_{2 n} \\
\vdots & \ddots & \vdots \\
-k_{N 1} \underline{\alpha}_{n 1} & \ldots & \sum_{j \in \mathcal{V}} k_{n j} \underline{\alpha}_{n j}
\end{array}\right]
$$

with

$$
k_{i j}= \begin{cases}1 & \text { if } j \in \mathcal{N}_{i} \\ 0 & \text { otherwise }\end{cases}
$$

By the Courant-Fisher Theorem and the fact that $\mathcal{L}_{\underline{\alpha}} \mathbf{1}=\mathbf{0}$, we have $\lambda_{2}\left(\mathcal{L}_{\underline{\alpha}}\right)=\inf _{x^{T} 1=0, x \neq 0} \frac{x^{T} \mathcal{L}_{\underline{\alpha}} x}{x^{T} x}$. Thus

$$
\begin{aligned}
& \dot{V}(x(t)) \leq-\lambda_{2}\left(\mathcal{L}_{\underline{\alpha}}\right) \cdot \tilde{x}^{T} \tilde{x} \\
& =-2 \lambda_{2}\left(\mathcal{L}_{\underline{\alpha}}\right) \sum_{i \in \mathcal{V}} \int_{x^{*}}^{x_{i}}\left(y-x^{*}\right) \mathrm{d} y \\
& \leq-2 \lambda_{2}\left(\mathcal{L}_{\underline{\alpha}}\right) \underline{\gamma} \sum_{i \in \mathcal{V}} \int_{x^{*}}^{x_{i}} \frac{y-x^{*}}{\gamma_{i}(y)} \mathrm{d} y=-2 \lambda_{2}\left(\mathcal{L}_{\underline{\alpha}}\right) \underline{\gamma} V(x) .
\end{aligned}
$$

By the comparison lemma [12], it follows that $V(x(t)) \leq$ $V(x(0)) \cdot e^{-2 \lambda_{2}\left(\mathcal{L}_{\underline{\alpha}}\right)} \underline{\underline{\gamma}}$. It is possible to express bounds of the convergence rate directly in terms of $\tilde{x}$. Observing that $\tilde{x}^{T} \tilde{x} \leq 2 \bar{\gamma} \sum_{i \in \mathcal{V}} \int_{x^{*}}^{x_{i}} \frac{y-x^{*}}{\gamma_{i}(y)} \mathrm{d} y$, and $V(x(0)) \leq \frac{1}{2 \underline{\gamma}} \tilde{x}^{T} \tilde{x}$, it follows that $\|\tilde{x}(t)\|_{2} \leq \sqrt{\frac{\bar{\gamma}}{\underline{\gamma}}}\|\tilde{x}(0)\|_{2} e^{-\lambda_{2}\left(\mathcal{L}_{\underline{\alpha}}\right) \underline{\gamma} t}$.

\section{CONSENSUS FOR DOUBLE INTEGRATOR DYNAMICS}

Consider the linear second-order dynamical system:

$$
\begin{aligned}
\dot{x}_{i} & =v_{i} \\
\dot{v}_{i} & =u_{i}
\end{aligned}
$$

where agent $i$ applies the following nonlinear consensus protocol:

$$
u_{i}=-\gamma_{i}\left(v_{i}\right) \sum_{j \in \mathcal{N}_{i}}\left[\alpha_{i j}\left(x_{i}-x_{j}\right)+\beta_{i j}\left(v_{i}-v_{j}\right)\right] .
$$

We show that under mild conditions, the consensus protocol (7) achieves asymptotic consensus.

Theorem 7. Consider the second order system (5)(6) under the consensus protocol (7), where $\alpha_{i j}(\cdot)$ and $\gamma_{i}(\cdot)$ satisfy assumptions 3 and 1 respectively, and $\beta_{i j}(\cdot)$ satisfies the same assumptions as $\alpha_{i j}(\cdot)$. The system achieves consensus with respect to $x$ and $v$, i.e. $\left|x_{i}-x_{j}\right| \rightarrow 0,\left|v_{i}-v_{j}\right| \rightarrow 0 \forall i, j \in \mathcal{G}$ as $t \rightarrow \infty$ for any initial condition $(x(0), v(0))$ if and only if $\mathcal{G}$ is connected. Furthermore, if consensus is reached, the velocities converge to a common value $\lim _{t \rightarrow \infty} v(t)=v^{*} 1$ satisfying $\sum_{i \in \mathcal{V}} \int_{0}^{v_{i}^{0}} \frac{1}{\gamma_{i}(y)} \mathrm{d} y=\int_{0}^{v^{*}} \sum_{i \in \mathcal{V}} \frac{1}{\gamma_{i}(y)} \mathrm{d} y$.

Remark 2. Theorem 7 generalizes both the literature on linear second-order consensus [22] as well as the literature on first-order nonlinear consensus [3]. By modifying the novel Lyapunov function as introduced in (4), we are able to prove that the agents reach consensus for the nonlinear 
consensus protocol also in the case of double integrator dynamics.

Proof: (Sufficiency:) We write (5)-(7) in vector form:

$$
\begin{aligned}
\dot{x} & =v \\
\dot{v} & =-\Gamma(v)[\mathcal{B} \alpha(\bar{x})+\mathcal{B} \beta(\bar{v})]
\end{aligned}
$$

where $\quad \alpha(\bar{x}) \quad=\quad\left[\alpha_{1}\left(\bar{x}_{1}\right), \ldots, \alpha_{m}\left(\bar{x}_{m}\right)\right]^{T}$, $\beta(\bar{x})=\left[\beta_{1}\left(\bar{x}_{1}\right), \ldots, \beta_{m}\left(\bar{x}_{m}\right)\right]^{T}, \quad m=|\mathcal{E}|$, and $\Gamma(x)=\operatorname{diag}\left(\left[\gamma_{1}\left(x_{1}\right), \ldots, \gamma_{n}\left(x_{n}\right)\right]\right)$. Consider the following candidate Lyapunov function, also used in [16]

$V(x, v)=\sum_{i \in \mathcal{V}}\left(\int_{v^{*}}^{v_{i}} \frac{y-v^{*}}{\gamma_{i}(y)} \mathrm{d} y+\frac{1}{2} \sum_{j \in \mathcal{N}_{i}} \int_{0}^{x_{i}-x_{j}} \alpha_{i j}(y) \mathrm{d} y\right)$.

consider:

$$
\begin{aligned}
\frac{\mathrm{d} E(v(t))}{\mathrm{d} t} & =\frac{\partial E}{\partial v} \frac{\partial v}{\partial t}=-\mathbf{1}^{T} \Gamma^{-1}(v) \Gamma(v)[B \alpha(\bar{x})+B \beta(\bar{v})] \\
& =-\mathbf{1}^{T} \mathcal{B} \alpha(\bar{x})-\mathbf{1}^{T} \mathcal{B} \beta(\bar{v})=0 .
\end{aligned}
$$

Thus we conclude that $\lim _{t \rightarrow \infty} x(t)=x^{*}(t) \mathbf{1}$ and $\lim _{t \rightarrow \infty} v(t)=v^{*} \mathbf{1}$ with $v^{*}$ given by the integral equation:

$$
\sum_{i \in \mathcal{V}} \int_{0}^{v_{i}^{0}} \frac{1}{\gamma_{i}(y)} \mathrm{d} y=\int_{0}^{v^{*}} \sum_{i \in \mathcal{V}} \frac{1}{\gamma_{i}(y)} \mathrm{d} y
$$

The existence and uniqueness of the solution to the above integral equation follows by from assumption 1 .

\section{EXAMPLE}

Consider a group of autonomous mobile agents in space.

By noting that $\frac{1}{2} \sum_{i \in \mathcal{V}} \sum_{j \in \mathbf{N}_{i}} x_{i}-x_{j}=\sum_{(i, j) \in \mathcal{E}} x_{i}-x_{j}$ we write $V(x, v)$ using the adjacency matrix $\mathcal{B}$ :

$V(x, v)=\int_{0}^{\bar{x}} \mathbf{1}^{T} \mathcal{B}^{T} \alpha(y) \mathrm{d} y+\int_{v^{*} \mathbf{1}}^{v} \tilde{y}^{T} \Gamma^{-1}(y) \mathbf{1} \mathrm{d} y \geq 0$

with $\tilde{y}=\left[\begin{array}{lll}y_{1}-v^{*} & \ldots & y_{n}-v^{*}\end{array}\right]^{T}$. Differentiating $V(x, v)$ with respect to time yields:

$$
\begin{aligned}
& \frac{d V(x, v)}{d t}=\frac{\partial V(x, v)}{\partial x} \frac{\partial x}{\partial t}+\frac{\partial V(x, v)}{\partial v} \frac{\partial v}{\partial t} \\
& =\alpha(\bar{x})^{T} \mathcal{B}^{T} v-\left(v-v^{*} \mathbf{1}\right)^{T} \Gamma^{-1}(v) \Gamma(v)[\mathcal{B} \alpha(\bar{x})+\mathcal{B} \beta(\bar{v})] \\
& =-v^{T} B \beta(\bar{v})+v^{*} \mathbf{1}^{T} \mathcal{B} \beta(\bar{v})=-\bar{v}^{T} \beta(\bar{v}) \leq 0
\end{aligned}
$$

with equality if and only if $\bar{v}=0$. We now invoke LaSalles invariance principle to show that the agreement point satisfies $\dot{v}=0$. The subspace where $\dot{V}(x, v)=0$ is given by $S_{1}=$ $\left\{(x, v) \mid v=v^{*}(t) \mathbf{1}\right\}$. Noting that on $S_{1}$ :

$$
\dot{v}=-\Gamma(v)[\mathcal{B} \alpha(\bar{x})+\mathcal{B} \beta(\bar{v})]=-\Gamma(v) \mathcal{B} \alpha(\bar{x}) \neq k(t) \mathbf{1} .
$$

To see this, suppose that $\dot{v}(t)=-\Gamma(v) \mathcal{B} \alpha(\bar{x})=k(t) \mathbf{1} \Leftrightarrow$ $\mathcal{B} \alpha(\bar{x})=\Gamma^{-1}(v) k(t) \mathbf{1}$, where $k(t) \neq 0$. Premultiplying by $\mathbf{1}^{T}$ yields $0=\mathbf{1}^{T} \mathcal{B} \alpha(\bar{x})=k(t) \mathbf{1}^{T} \Gamma^{-1}(v) \mathbf{1} \neq 0$, which is a contradiction since $k(t) \neq 0$ by assumption. Hence $S_{1}$ only contains trajectories where $v=v^{*} \mathbf{1}, \dot{v}=0$. This also implies that no trajectory where $x \neq x^{*}(t) \cdot \mathbf{1}$ can lie on $S_{1}$, since $v=v^{*} \mathbf{1}$ implies $0=\dot{v}=-\Gamma(v) \mathcal{B} \alpha(\bar{x})$, which implies $\mathcal{B} \bar{x}=0 \Rightarrow \mathcal{L} x=0 \Rightarrow x=x^{*}(t) \mathbf{1}$ since $\mathcal{G}$ is connected. Thus the agents converge to a moving point in $\mathbb{R}$, $\left|x_{i}-x_{j}\right| \rightarrow 0,\left|v_{i}-v_{j}\right| \rightarrow 0 \forall i, j \in \mathcal{G}$ as $t \rightarrow \infty$ and furthermore $\dot{v}(t)=0$.

(Necessity:) Assume that $\mathcal{G}$ is disconnected. Then there exist two connected components, $\mathcal{G}_{1}, \mathcal{G}_{2} \in \mathcal{G}$, such that $\mathcal{V}\left(\mathcal{G}_{1}\right) \cap \mathcal{V}\left(\mathcal{G}_{2}\right)=\emptyset$ and there is no edge between $\mathcal{G}_{1}$ and $\mathcal{G}_{2}$. Let $x_{i}(0)=x_{0}^{1}, v_{i}(0)=v_{0}^{1} \forall i \in \mathcal{G}_{1}$ and $x_{i}(0)=$ $x_{0}^{2}, v_{i}(0)=v_{0}^{2} \forall i \in \mathcal{G}_{2}$, where $v_{0}^{1} \neq v_{0}^{2}$. Since $\mathcal{G}_{1}$ and $\mathcal{G}_{2}$ are vertex-disjoint and there is no edge connecting $\mathcal{G}_{1}$ and $\mathcal{G}_{2}, \dot{v}_{i}=0 \forall i \in \mathcal{V}\left(\mathcal{G}_{1}\right) \cup \mathcal{V}\left(\mathcal{G}_{2}\right)$. Thus consensus cannot be reached. Next we show that $E(v)=\sum_{i \in \mathcal{V}} \int_{0}^{v_{i}} \frac{1}{\gamma_{i}(y)} \mathrm{d} y=$ $\int_{0}^{v} \mathbf{1}^{T} \Gamma^{-1}(v) \mathbf{1} \mathrm{d} y$ is invariant under the protocol (7). Indeed,
The agents are denoted $v_{1}, \ldots, v_{5}$, whose communication

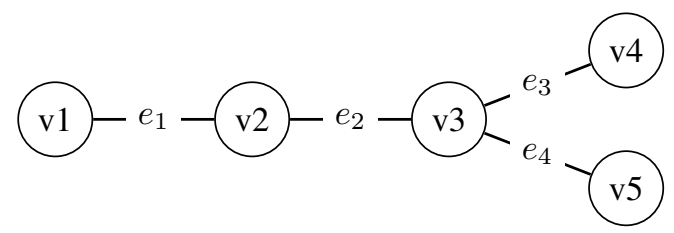

Figure 1. Communication topology of the agents.

topology is given by the undirected graph in figure 1 . The goal is to reach consensus in one dimension by applying a distributed consensus control law by using only relative position and velocity measurements. The raw control signal is the power applied to the agent's engine, $P_{i}$. However, the acceleration in an observers reference frame is $a_{i}=\frac{P_{i}}{\left|v_{i}\right|}$, where $v_{i}$ is the agents velocity. We assume that the agents only have access to relative measurements, and hence are unaware of their absolute positions. This scenario can be modeled by our proposed nonlinear consensus protocol (7), where the gain function $\gamma_{i}(y)=\frac{1}{|y|+c} \quad \forall i \in \mathcal{V}$ captures the dependence of the agents acceleration on it's absolute speed. $c \in \mathbb{R}^{+}$is arbitrarily small, and ensures the boundedness of $\gamma_{i}$. The interaction functions are chosen to be $\alpha_{i j}(y)=2 \beta_{i j}(y)=20\left(e^{y}-1\right) \operatorname{sgn}(y) \quad \forall(i, j) \in \mathcal{E}$. It is clear that this situation cannot be modeled by any previously proposed linear consensus protocols. Figures 2 and 3 show the state trajectories for different initial conditions. Due to theorem 7, consensus is reached, and the final consensus velocity, as seen from an observer, can be calculated and is shown with a dashed lines.

\section{CONCLUSIONS AND FUTURE WORK}

In this paper we have considered a class of nonlinear consensus protocols for first-order and second-order dynamics. Necessary and sufficient conditions for consensus were derived for static communication topologies under single and double integrator dynamics, and for switching under single integrator dynamics. In all cases, expressions for the convergence points were specified. Necessary and sufficient conditions for the convergence were derived for static directed 

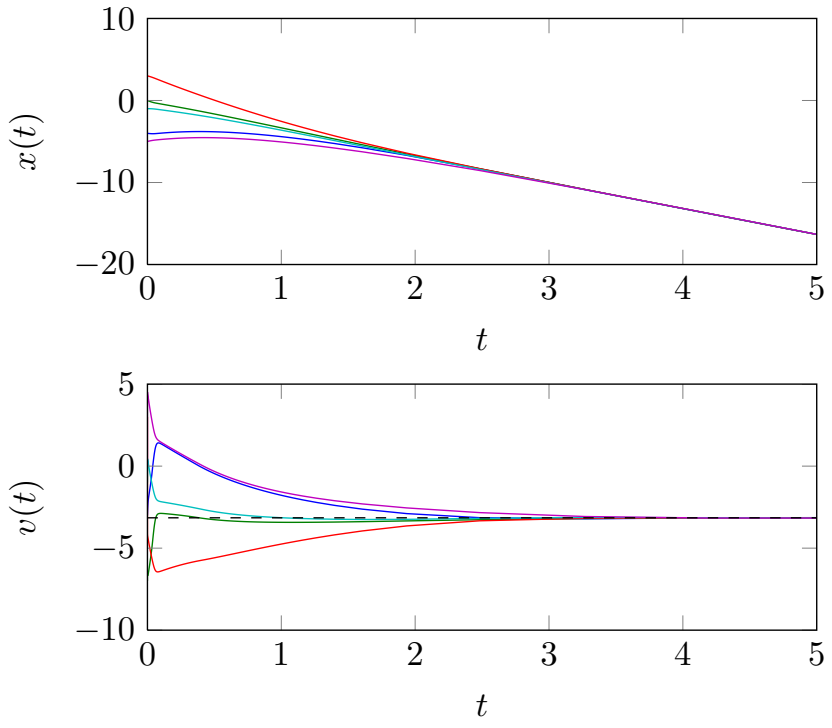

Figure 2. State trajectories with $x(0)=[-4,0,3,-1,-5], v(0)=$ $[-3,-7,3,-1,0]^{T}$
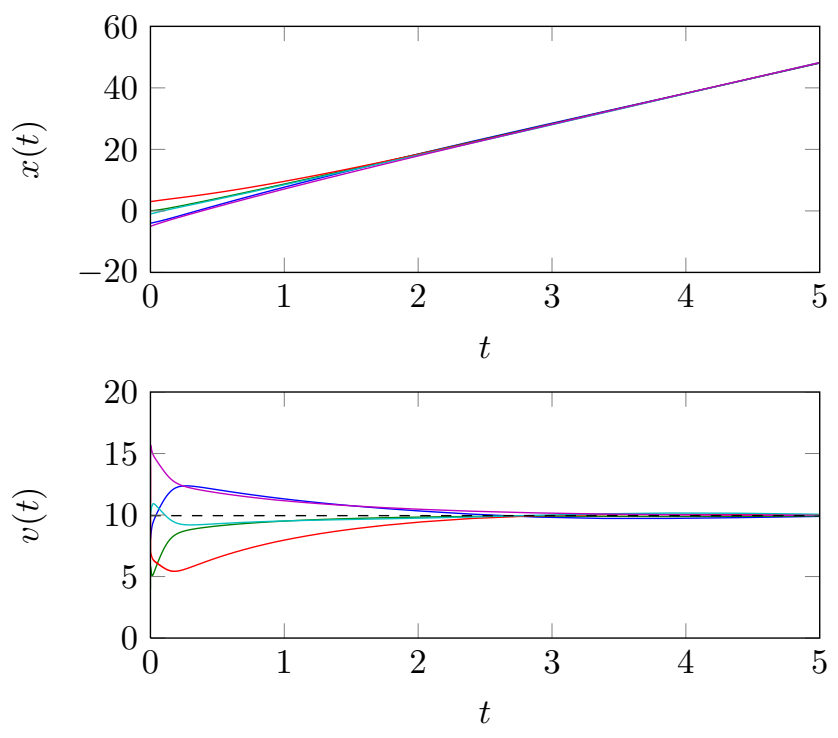

Figure 3. State trajectories with $x(0)=[-4,0,3,-1,-5], v(0)=$ $[8,4,14,10,11]^{T}$

communication under single integrator dynamics. For static communication topologies under single integrator dynamics, we derived bounds on the exponential convergence using a novel Lyapunov function.

Possible applications could include consensus problems with preferred and non-preferred absolute agreement points. The state-dependent convergence speed could also be used to capture inherent properties of the agents dynamics, as demonstrated in the example. Other possible applications include distributed estimation with state-dependent sensor noise and measurement range.

\section{ACKNOWLEDGEMENTS}

The authors would like to thank Guodong Shi for his useful suggestions.

\section{REFERENCES}

[1] A. Ajorlou, A. Momeni, and A.G. Aghdam. Sufficient conditions for the convergence of a class of nonlinear distributed consensus algorithms. Automatica, 47(3):625 - 629, 2011.

[2] D. Bauso, L. Giarre, and R. Pesenti. Mechanism design for optimal consensus problems. pages $3381-3386$, dec. 2006.

[3] D. Bauso, L. Giarre, and R. Pesenti. Non-linear protocols for optimal distributed consensus in networks of dynamic agents. Systems \& Control Letters, 55(11):918 - 928, 2006.

[4] F. Bullo, J. Cortés, and S. Martínez. Distributed Control of Robotic Networks. Applied Mathematics Series. Princeton University Press, 2009.

[5] F. Chen, Z. Chen, L. Xiang, Z. Liu, and Z. Yuan. Reaching a consensus via pinning control. Automatica, 45(5):1215 - 1220, 2009.

[6] J. Cortés. Analysis and design of distributed algorithms for xconsensus. In IEEE Conference on Decision and Control, pages 3363 -3368 , dec. 2006.

[7] Cortés, J. Distributed algorithms for reaching consensus on general functions. Automatica, 44(3):726 - 737, 2008.

[8] D.V. Dimarogonas and K.J. Kyriakopoulos. Connectedness preserving distributed swarm aggregation for multiple kinematic robots. IEEE Transactions on Robotics, 24(5):1213 -1223, oct. 2008.

[9] J.A. Fax and R.M. Murray. Information flow and cooperative control of vehicle formations. IEEE Transactions Automatic Control, 49(9):1465 - 1476, sept. 2004.

[10] Q. Hui and W.M. Haddad. Distributed nonlinear control algorithms for network consensus. Automatica, 44(9):2375 - 2381, 2008.

[11] M. Ji and M. Egerstedt. Distributed coordination control of multiagent systems while preserving connectedness. IEEE Transactions on Robotics, 23(4):693 -703, aug. 2007.

[12] H.K. Khalil. Nonlinear Systems. Prentice Hall, 2002.

[13] Z. Lin, B. Francis, and M. Maggiore. State agreement for continuoustime coupled nonlinear systems. SIAM Journal on Control and Optimization, 46:288-307, March 2007.

[14] Liu, Y. and Passino, K.M. and Polycarpou, M.M. Stability analysis of w-dimensional asynchronous swarms with a fixed communication topology. IEEE Transactions Automatic Control, 48:76-95, 2003.

[15] L. Moreau. Stability of multiagent systems with time-dependent communication links. IEEE Transactions on Automatic Control, 50(2): 169 - 182, feb. 2005.

[16] U. MuľL andnz, A. Papachristodoulou, and F. AllgoÎ́ andwer. Robust consensus controller design for nonlinear relative degree two multiagent systems with communication constraints. Automatic Control, IEEE Transactions on, 56(1):145 -151, jan. 2011.

[17] R. Olfati, S. Richard, and R.M. Murray. Consensus protocols for networks of dynamic agents. In American Control Conference, pages 951-956, 2003.

[18] R. Olfati-Saber. Flocking for multi-agent dynamic systems: Algorithms and theory. IEEE Transactions on Automatic Control, 51(3):401 - 420, march 2006.

[19] R. Olfati-Saber, J.A. Fax, and R.M. Murray. Consensus and cooperation in networked multi-agent systems. Proceedings of the IEEE, 95(1):215 -233, jan. 2007.

[20] R. Olfati-Saber and R.M. Murray. Consensus problems in networks of agents with switching topology and time-delays. IEEE Transactions on Automatic Control, 49(9):1520 - 1533, sept. 2004.

[21] W. Ren. On consensus algorithms for double-integrator dynamics. IEEE Transactions on Automatic Control, 53(6):1503 -1509, july 2008

[22] W. Ren and R.W. Beard. Distributed Consensus in Multi-vehicle Cooperative Control. Springer, 2008.

[23] H.G. Tanner, A. Jadbabaie, and G. J. Pappas. Flocking in fixed and switching networks. IEEE Transactions on Automatic Control, 52(5):863 -868, may 2007.

[24] H.G. Tanner, A. Jadbabaie, and G.J. Pappas. Stable flocking of mobile agents, part i: Fixed topology. In IEEE Conference on Decision and Control, volume 2, pages 2010 - 2015 Vol.2, dec. 2003.

[25] W Yu, G Chen, and M. Cao. Some necessary and sufficient conditions for second-order consensus in multi-agent dynamical systems. Automatica, 46(6):1089 - 1095, 2010. 